\title{
Perceived Level of Stress, \& Coping Strategies among Saudi Nursing Student
}

\author{
Luma Ghazi Al Zamil \\ Nursing College, Umm Al Qura University, Makkal Al Mukaramah, UKA
}

\begin{abstract}
The present study aimed at assessing the stress level\& determine the coping strategies among the nursing students at umm al qura university. The study design was descriptive, cross- sectional. The study was conducted at the beginning of second semester inthe academic year 2015/2016. The sample consisted of three tools for data collection: which included a socio-demographicprofile sheet, two scales; Perceived Stress Scale14(PSS 14) to assess stress level, and The Brief Coping Orientation for Problem Experiences (Brief COPE) to assess The Students' coping strategies. Results revealed have that the percentage of stress level was moderate in students. Adaptive stress-coping strategies were more frequently used among a sample of nursing student at umm al Qura university in Saudi Arabia. The study recommended that It is important for nurse educator to find out the sources of stress and coping strategies used by the students so that they can be helped to cope well with upcoming problems and situations.
\end{abstract}

Key words: Stress, Coping Strategies, stressors (socio-demographic) \&Nursing Saudi Students.

\section{Introduction}

Stress refers to a dynamic interaction between the individual and the environment ${ }^{[1]}$. This interaction may lead to cognitive, emotional and behavioral alteration ${ }^{[2]}$.nursing students also have stress related to their educational studies and personal/social experience ${ }^{[3]}$.Common sources of stress in nursing students basically include: a) academic-related concerns such as workload, lack of knowledge, examination, teaching style; b) clinical practice for example, lack of competence, taking care of death and dying, shifting, supervision, assessment; c) relationships with others (clients, peers, teachers or other health professionals) ${ }^{[4]}$; and d) personal factors such as gender, age, new surroundings away from home, family structure, financial burdens and job roles [3]:[5]; [6]. Students may experience stress in different fields, therefore; the insufficiency of theoretical training, lack of skills in clinical practices, to take the responsibility of the patient, time pressure, lack of motivation and accommodation, social life, relationship with the opposite sex, new responsibilities, living in a new environment may be perceived as stress ${ }^{[7,8,9,10,11,12,13]}$. Sources of stress also vary in accordance with the students' years. It has been stated that third year students' level of stress is higher ${ }^{[14]}$, and the senior year students' depression mark averages are higher ${ }^{[15]}$.

\section{Aim of study:}

The aim of my study to assess the perceived stress level \& coping strategies among nursing students at Umm Al Qura University.

\section{Objectives:}

$\checkmark$ To assess the stress level among the nursing students at umm al Qura University.

$\checkmark$ To determine the coping strategies that the nursing student can be applied to decrease the stressors.

\subsection{Study design :}

\section{Methods:}

The study was a descriptive, cross- sectional design was using a self administrated questionnaires for data collection

\subsection{Setting \& Sampling:}

The study took place at nursing faculty in umm al qua university. All students study in nursing program was recruited to minimize the sampling errors. The total number of nursing students was 107 excluding 10 students who participated in the instrument test - retest reliability study, there were 97 students participates in main study.

\subsection{Ethical consideration:}

Approval was obtain from the research ethics committee of the study setting before conducting the study. Permission for using the questionnaires was granted by original responding author .All participates were assured for anonymity \& confidentiality. 


\subsection{Measurements/ instruments:}

Data collection instrument included the demographic profile, The Brief COPE, \&The Perceived Stress Scale (PSS). The Brief COPE: it is a 28-item self-administered inventory containing 14 subscales of two items each that aims to assess how individuals cope with stress in daily life. The 14 subscales include: active coping, planning, positive reframing, acceptance, humor, religion, using emotional support, using instrumental support, self-distraction, denial, venting, substance use, behavioral disengagement, and self-blame. Each item asked participants to rate on a 4-point Likert scale ranging from 1(I don't do this at all) to 4( I do this a lot ). Since, there are two groups coping style, the current study, adaptive and maladaptive coping styles are used. Adaptive coping include active coping, planning, positive reframing, acceptance, humor, religion, using emotional support, and using instrumental support. While maladaptive coping consists of self-distraction, denial, venting, substance use, behavioral disengagement, and self-blame ${ }^{[16]}$.Scale are computed as follows (with no reversals of coding): active coping ( items 2\&7), planning ( items 14\&25), positive reframing( items 12\&17), acceptance( items 20\&24), humor( items 18\&28), religion( items 22\&27), using emotional support (items 5\&15), selfdistraction (items $1 \& 19$ ), denial(items 3\&8), venting( items 9\&21), substance use(items 4\&11), behavioral disengagement( items 6\&16), self-blame( items 13\&26) and using instrumental support( items 10\&23) [17]

Brief COPE Scale (Arabic, Malay\& English Version) confirms fairly good reliability and validity. ${ }^{[\mathbf{1 8 , 1 9 \& 2 0}]}$

The Perceived Stress Scale :It includes ten Items which were designed to tap how unpredictable, uncontrollable, and overloaded respondents find their lives.Responses were assessed on a 5 point scale, with '1' $=$ 'never' and '5' = 'very often'.(Most of time/ Very Often) 5 degrees, (Often/ Fairly Often) 4 degrees, (Sometimes) 3 degrees, (Hardly/ Almost Never) two degrees, (Never) one degree. The scale also includes a number of direct queries about current levels of experienced stress. Scale scores are obtained by reversing responses (e.g., $0=4,1=3,2=2,3=1 \& 4=0$ ) to the four positively stated items (items $4,5,7, \& 8$ ) and then summing across all scale items. Individual scores on PSS can range from 0 to 40 with higher scores indicating

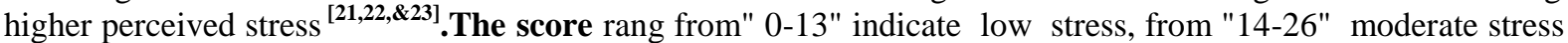
and from "27-40" high perceived stress ${ }^{[24]}$. The Arabic version Perceived Stress Scale reliability and validity were evaluated. The Cronbach's alpha coefficients were 0.74 (Factor 1), 0.77 (Factor 2) and 0.80 for the Arabic version Perceived Stress Scale overall. The test-retest reliability had an intra-correlation coefficient of 0.90. ${ }^{[25]}$

\subsection{Data collection /procedure :}

Completion of the questionnaire was voluntary and would not affect the students' progress in the college . A face-to-face session was held with the students. Data was collected by guided self-administered questionnaire. The time taken by the students for filling in the questionnaire was around 15 minutes. The questionnaires were collected on the same day.

\subsection{Data analysis:}

The data will analyze using the Statistical Package for Social Sciences (SPSS) Version 19. Descriptive statistics was used to calculate means and frequencies of the respondents according to specific demographic profile variables. Proper statistical test Correlation Coefficient(chi square "Fisher's Exact Test" )was used to estimate the statistical significant differences and to examine demographic variable on stress, \&coping. P. value will considered significant at P. $<0.05$ or less. This statistics was used to test the hypothesis of relationship between paired variables.

III. Results

Table (1) Socio - demographic characteristics

\begin{tabular}{|c|c|c|c|}
\hline \multirow{2}{*}{ Items } & Variables & No. & $\%$ \\
\hline \multirow{4}{*}{ Age } & $19-20$ & 56 & 57.7 \\
\cline { 2 - 4 } & $21-22$ & 34 & 35.1 \\
\cline { 2 - 4 } & $23-24$ & 3 & 3.1 \\
\cline { 2 - 4 } & $>24$ & 4 & 4.1 \\
\hline Variables & $M$ & SD & Range \\
\hline Age & 1.54 & .751 & $19-24$ \\
\hline marriage status & single & 89 & 91.8 \\
\cline { 2 - 4 } & married & 7 & 7.2 \\
\cline { 2 - 4 } & divorce & 1 & 1.0 \\
\hline \multirow{3}{*}{ No. Of children of the students } & Ochild & 93 & 95.9 \\
\cline { 2 - 4 } & Ichild & 2 & 2.1 \\
\cline { 2 - 4 } & 2 child & 1 & 1.0 \\
\cline { 2 - 4 } & 3child & 1 & 1.0 \\
\hline Year of study & second year & 59 & 60.8 \\
\cline { 2 - 4 } & third year & 15 & 15.5 \\
\cline { 2 - 4 } & fourth year & 23 & 23.7 \\
\hline Living with family & yes & 93 & 95.9 \\
\hline
\end{tabular}




\begin{tabular}{|c|c|c|c|}
\hline & no & 4 & 4.1 \\
\hline Breadwinner for students & father & 70 & 72.2 \\
\cline { 2 - 4 } & mother & 8 & 8.2 \\
\cline { 2 - 4 } & brother & 3 & 3.1 \\
\cline { 2 - 4 } & husband & 5 & 5.2 \\
\cline { 2 - 4 } & other & 11 & 11.3 \\
\hline
\end{tabular}

\subsection{Demographic characteristics of participations:}

Through reviewing the results illustrated in the table above, shows the following:

I. According to the age: the results illustrates that $57.7 \%$ from the total of the nursing students are 19-20 years old and they are the majority of the study sample. While $35.1 \%$ from the total of the nursing students are $21-22$ years old, also found that $3.1 \%$ from the total of the nursing students are 23-24 years old, and finally $4.1 \%$ from the total of the nursing study are above 24 years old .

II. According to marriage status : the results revealed that the majority from the total of the nursing students $(91.8 \%)$ are single .While $7.2 \%$ are married $\& 1.0 \%$ are divorce.

III. According to the number Of children of the students : the results shows that $95.9 \%$ haven't children since $91.8 \%$ are single. While $2.1 \%$ have one children $\& 1.0 \%$ have two or three children .

IV. According to Living with family: the results clarify that $95.9 \%$ of students living with family and they are the majority of the study sample.

V. According to Breadwinner for students : the results revealed that the majority breadwinner for students $72.2 \%$ are father . while $11.3 \%$ are other institution, also found that $8.2 \%$ are mother, $5.2 \%$ are husband \& $3.1 \%$ are brother .

\subsection{Stress level Among Nursing Students:}

Findings revealed that $75.3 \%$ from the total of nursing students had moderate stress. Whereas $19.6 \%$ are high stress, \& $5.2 \%$ are low stress. Also the results illustrate that $87.0 \%$ of fourth year nursing student has moderate stress, whereas $73.3 \%$ of third year $\& 71.2 \%$ of the second year. Figure (1) illustrates this:

Figure (1) :Stress level Among Nursing Students

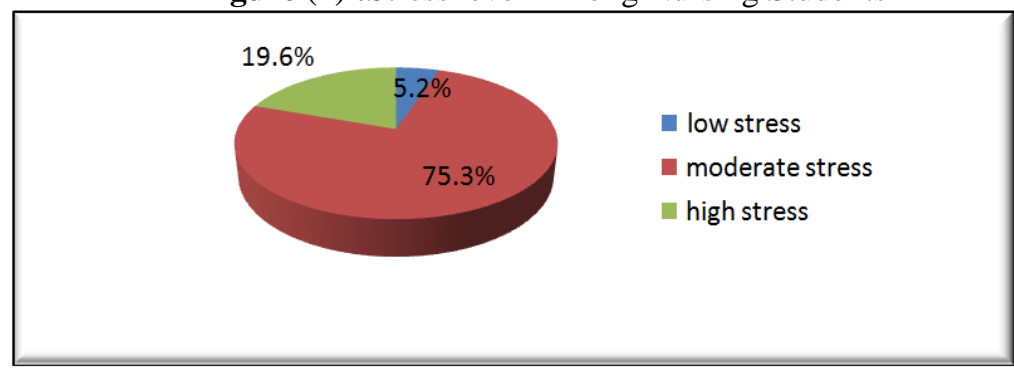

Table (2) Stress Level Based on Socio Demographic Changes n=96

\begin{tabular}{|c|c|c|c|c|c|c|}
\hline Socio - demographic & Categories & $\begin{array}{c}\text { Low stress } \\
\text { No. }(\%)\end{array}$ & $\begin{array}{c}\text { Moderate stress } \\
\text { No. }(\%) \\
\end{array}$ & $\begin{array}{c}\text { High stress } \\
\text { No. }(\%)\end{array}$ & $\begin{array}{c}\text { Fisher's } \\
\text { Exact Test }\end{array}$ & $\begin{array}{c}\text { Chi square } \\
P \text {-value }\end{array}$ \\
\hline \multirow[t]{5}{*}{ Age } & $19-20$ & $5(8.9)$ & $37(66.1)$ & $14(25.0)$ & \multirow{5}{*}{.287} & \multirow[t]{5}{*}{.090} \\
\hline & $21-22$ & $\mathbf{0}$ & $29(85.3)$ & $5(14.7)$ & & \\
\hline & $23-24$ & $\mathbf{0}$ & $3(100)$ & $\mathbf{0}$ & & \\
\hline & $>24$ & $\mathbf{0}$ & $4(100)$ & $\mathbf{0}$ & & \\
\hline & Total & $5(5.2)$ & $73(75.3)$ & 19(19.6) & & \\
\hline \multirow[t]{4}{*}{ marriage status } & single & $5(5.7)$ & 65(73.9) & $18(20.5)$ & \multirow{4}{*}{1.000} & \multirow{4}{*}{.265} \\
\hline & married & $\mathbf{0}$ & $6(85.7)$ & $1(14.3)$ & & \\
\hline & divorce & $\mathbf{0}$ & $1(100)$ & $\mathbf{0}$ & & \\
\hline & Total & $5(5.2)$ & $72(75.0)$ & 19(19.8) & & \\
\hline \multirow{5}{*}{$\begin{array}{l}\text { No. Of children of } \\
\text { the students }\end{array}$} & Ochild & $5(5.4)$ & $70(75.3)$ & $18(19.4)$ & \multirow{5}{*}{.515} & \multirow{5}{*}{.132} \\
\hline & 1child & $\mathbf{0}$ & $2(100)$ & $\mathbf{0}$ & & \\
\hline & 2 child & $\mathbf{0}$ & 1(100) & $\mathbf{0}$ & & \\
\hline & 3 child & $\mathbf{0}$ & $\mathbf{0}$ & $1(100)$ & & \\
\hline & Total & $5(5.2)$ & $73(75.3)$ & 19(19.6) & & \\
\hline \multirow[t]{4}{*}{ Year of study } & second year & 3(5.1) & 42(71.2) & 14(23.7) & \multirow{4}{*}{.313} & \multirow{4}{*}{.079} \\
\hline & third year & 2(13.3) & 11(73.3) & $2(13.3)$ & & \\
\hline & fourth year & $\mathbf{0}$ & $20(87.0)$ & 3(13.0) & & \\
\hline & Total & $5(5.2)$ & $73(75.3)$ & 19(19.6) & & \\
\hline \multirow[t]{4}{*}{ Living with family } & yes & $5(5.4)$ & $70(75.3)$ & $18(19.4)$ & \multirow[t]{3}{*}{1.000} & \multirow[t]{3}{*}{.359} \\
\hline & no & $\mathbf{0}$ & $3(75.0)$ & $1(25.0)$ & & \\
\hline & Total & $5(5.2)$ & $73(75.3)$ & 19(19.6) & & \\
\hline & father & $4(5.7$ & 49(70.0) & 17(24.3) & .501 & $.032 *$ \\
\hline
\end{tabular}




\begin{tabular}{|c|c|c|c|c|}
\hline \multirow{5}{*}{$\begin{array}{l}\text { Breadwinner for } \\
\text { students }\end{array}$} & mother & $1(12.5)$ & $6(75.0)$ & $1(12.5)$ \\
\hline & brother & 0 & $3(\mathbf{1 0 0})$ & 0 \\
\hline & husband & 0 & $4(80.0)$ & $1(20.0)$ \\
\hline & other & $\mathbf{0}$ & $11(100)$ & 0 \\
\hline & Total & $5(5.2)$ & $73(75.3)$ & $19(19.6)$ \\
\hline
\end{tabular}

$\mathrm{p}^{*<0.05 \text {, significant }}$

1.9. The relation between the demographic characteristic \& level of stress. Through reviewing the results illustrated in the table 2 , it shows :

That there is a significant correlation between the nursing students' stress level and Breadwinner for students ( $\mathrm{p}=0.032$ ), as it shows that other institution \& brother as breadwinner for student has $100 \%$ moderate stress levelcomparing with the other breadwinner categories. While the results indicates that there is no correlation between the stress level and the education of age $(\mathrm{p}=0.090)$, the marriage status $(\mathrm{p}=0.265)$. , No. Of children for the students $(\mathrm{p}=0.132)$, Year of study $(\mathrm{p}=0.079)$, \&Living with family $(\mathrm{p}=0.359)$.

Figure (2) copies strategies among nursing students.

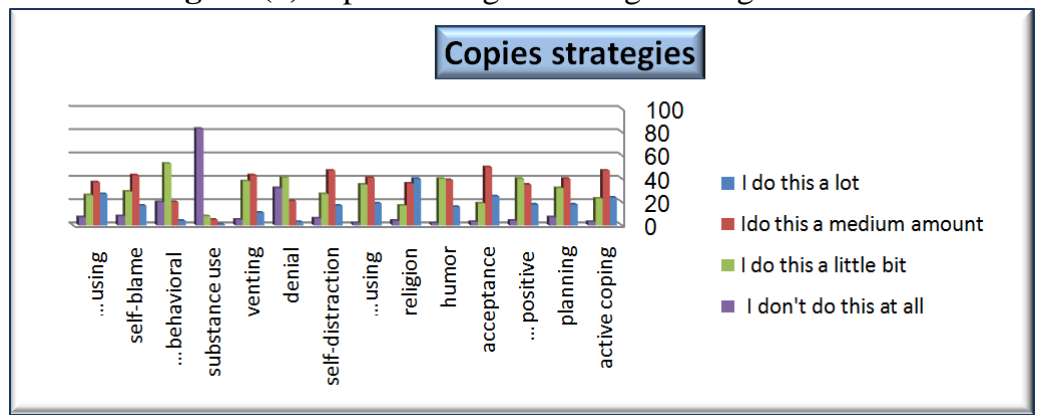

1.10. Coping strategies used by students :

Figure 2 shows that: The results showed that $41.2 \%$ nursing students do a lot of (religion) as copy strategy, while do a moderate amount $51.5 \%$ of acceptance, $48.5 \%$ of active coping \& $48.5 \%$ of self distraction. whereas , $42.3 \%$ nursing students do a little bit of denial $\& 41.2 \%$ of positive reframing\& humor as copy strategy. but also , the result revealed that $85.6 \%$ of nursing student don't do the substance use as copy strategy and they are the minimum used as the study sample.

Table (3) Frequency of copies strategies used by nursing students $n=97$

\begin{tabular}{|c|c|c|c|c|c|c|c|c|c|c|c|c|c|}
\hline \multirow{2}{*}{$\begin{array}{c}\text { Socio- } \\
\text { demographic }\end{array}$} & \multirow[t]{2}{*}{ Categories } & \multicolumn{4}{|c|}{ Active Coping } & \multicolumn{4}{|c|}{ Planning } & \multicolumn{4}{|c|}{ Positive Reframing } \\
\hline & & $\begin{array}{l}\text { I don't } \\
\text { do this } \\
\text { at allN }\end{array}$ & $\begin{array}{c}\text { I do this a } \\
\text { little bit } \\
N\end{array}$ & $\begin{array}{c}\text { Ido this a } \\
\text { medium } \\
\text { amount } \\
N\end{array}$ & $\begin{array}{c}\text { Ido this a } \\
\text { lot } \\
N\end{array}$ & $\begin{array}{c}\text { I don't do } \\
\text { this at all } \\
N\end{array}$ & $\begin{array}{c}\text { I do this a } \\
\text { little bit } \\
N\end{array}$ & $\begin{array}{c}\text { I do this a } \\
\text { medium } \\
\text { amount } \\
N\end{array}$ & $\begin{array}{c}\text { I do this a } \\
\text { lot } \\
N\end{array}$ & $\begin{array}{c}\text { I don't do } \\
\text { this at all } \\
N\end{array}$ & $\begin{array}{l}\text { I do this a } \\
\text { little bit } \\
N\end{array}$ & $\begin{array}{l}\text { Id do this a } \\
\text { medium } \\
\text { amount } \\
N\end{array}$ & $\begin{array}{c}\text { I do this a } \\
\text { lot } \\
N\end{array}$ \\
\hline \multirow[t]{4}{*}{ Age } & $19-20$ & 1(1) & $12(12.5)$ & $29(30.2)$ & $14(14.58)$ & $5(5.20)$ & $14(14.58)$ & $27(28.12)$ & $9(9.37)$ & 1(1) & $23(23.95)$ & $23(23.95)$ & $9(9.37)$ \\
\hline & $21-22$ & $2(2)$ & $10(10.4)$ & $15(15.62)$ & $7(7.29)$ & $2(2)$ & $15(15.62)$ & $12(12.5)$ & $5(5.20)$ & $3(3)$ & $15(15.62)$ & $8(8.33)$ & $8(8.33)$ \\
\hline & $23-24$ & $0(0)$ & $0(0)$ & $3(3)$ & $O(0)$ & $0(0)$ & $1(1)$ & $1(1)$ & $1(1)$ & $0(0)$ & $0(0)$ & $2(2)$ & $1(1)$ \\
\hline & $>24$ & $O(0)$ & $1(1)$ & $O(0)$ & $3(3)$ & $0(0)$ & $1(1)$ & $O(0)$ & $3(3)$ & $0(0)$ & $2(2)$ & $2(2)$ & $0(0)$ \\
\hline \multirow[t]{3}{*}{ marriage status } & single & $3(3)$ & $21(21.87)$ & 43 & $21(21.87)$ & $7(7.29)$ & $27(28.12)$ & $38(39.58)$ & $15(15.62)$ & $4(4.16)$ & $33(34.37)$ & $33(34.37)$ & $18(18.75)$ \\
\hline & married & $0(0)$ & $2(2)$ & $3(3)$ & $2(2)$ & $0(0)$ & $4(4.16)$ & $0(0)$ & $3(3)$ & $0(0)$ & $6(6.25)$ & $1(1)$ & $0(0)$ \\
\hline & divorce & $O(0)$ & $0(0)$ & $1(1)$ & $0(0)$ & $0(0)$ & $0(0)$ & $1(1)$ & $0(0)$ & $0(0)$ & $0(0)$ & $1(1)$ & $0(0)$ \\
\hline \multirow{4}{*}{$\begin{array}{l}\text { No. Of children of } \\
\text { the students }\end{array}$} & Ochild & $3(3)$ & $23(23.95)$ & $45(46.87)$ & $22(22.91)$ & $7(7.29)$ & $30(31.25)$ & $40(41.66)$ & $15(15.62)$ & $4(4.16)$ & $37(38.54)$ & $34(35.41)$ & $18(18.75)$ \\
\hline & Ichild & $0(0)$ & $0(0)$ & $1(1)$ & $1(1)$ & $0(0)$ & $1(1)$ & $0(0)$ & $1(1)$ & $0(0)$ & $2(2)$ & $0(0)$ & $0(0)$ \\
\hline & 2 child & $0(0)$ & $O(0)$ & $O(0)$ & $1(1)$ & $O(0)$ & $0(0)$ & $0(0)$ & $1(1)$ & $0(0)$ & $1(1)$ & $0(0)$ & $0(0)$ \\
\hline & 3child & $O(0)$ & $O(0)$ & $1(1)$ & $O(0)$ & $0(0)$ & $O(0)$ & $0(0)$ & $1(1)$ & $O(0)$ & $0(0)$ & $1(1)$ & $0(0)$ \\
\hline \multirow[t]{3}{*}{ Year of study } & second year & $2(2)$ & $13(13.54)$ & $28(29.16)$ & $16(16.66)$ & $6(6.25)$ & $16(16.66)$ & $25(26.04)$ & $11(11.45)$ & $2(2)$ & $25(26.04)$ & $23(23.95)$ & $9(9.37)$ \\
\hline & third year & $O(0)$ & $5(5.20)$ & $6(6.25)$ & $4(4.16)$ & $0(0)$ & $6(6.25)$ & $8(8.33)$ & $1(1)$ & $0(0)$ & $6(6.25)$ & $5(5.20)$ & $4(4.16)$ \\
\hline & fourth year & $1(1)$ & $5(5.20)$ & $13(13.54)$ & $4(4.16)$ & $1(1)$ & $9(9.37)$ & $7(7.29)$ & $6(6.25)$ & $2(2)$ & $9(9.37)$ & $7(7.29)$ & $5(5.20)$ \\
\hline \multirow{2}{*}{$\begin{array}{c}\text { Living with } \\
\text { family }\end{array}$} & yes & $3(3)$ & $22(22.91)$ & $44(45.83)$ & $24(25)$ & $6(6.25)$ & $30(31.25)$ & $39(40.62)$ & $17(17.7)$ & $4(4.16)$ & $38(39.58)$ & $34(35.41)$ & $17(17.7)$ \\
\hline & no & $O(0)$ & $1(1)$ & $3(3)$ & $0(0)$ & $1(1)$ & $1(1)$ & $1(1)$ & $1(1)$ & $0(0)$ & $2(2)$ & $1(1)$ & $1(1)$ \\
\hline \multirow{5}{*}{$\begin{array}{l}\text { Breadriinner for } \\
\text { students }\end{array}$} & father & $3(3)$ & $16(16.66)$ & $36(37.5)$ & $15(15.62)$ & $6(6.25)$ & $22(22.91)$ & $31(32.29)$ & $11(11.45)$ & $2(2)$ & $26(27.08)$ & $27(28.12)$ & $15(15.62)$ \\
\hline & mother & $0(0)$ & $2(2)$ & $4(4.16)$ & $2(2)$ & $0(0)$ & $1(1)$ & $3(3)$ & $3(3)$ & $2(2)$ & $2(2)$ & $3(3)$ & $1(1)$ \\
\hline & brother & $0(0)$ & $1(1)$ & $2(2)$ & $0(0)$ & $0(0)$ & $3(3)$ & $0(0)$ & $0(0)$ & $0(0)$ & $1(1)$ & $2(2)$ & $0(0)$ \\
\hline & husband & $0(0)$ & 1(1) & $2(2)$ & $2(2)$ & $0(0)$ & $2(2)$ & $0(0)$ & $3(3)$ & $0(0)$ & $4(4.16)$ & $1(1)$ & $0(0)$ \\
\hline & other & $0(0)$ & $3(3)$ & $3(3)$ & $5(5.20)$ & 1(1) & $3(3)$ & $6(6.25)$ & $1(1)$ & $0(0)$ & $7(7.29)$ & $2(2)$ & $2(2)$ \\
\hline
\end{tabular}


Perceived Level Of Stress, \& Coping Strategies Among Saudi Nursing Student

\begin{tabular}{|c|c|c|c|c|c|c|c|c|c|c|c|c|c|}
\hline \multirow{2}{*}{$\begin{array}{c}\text { Socio- } \\
\text { demographic }\end{array}$} & \multirow[t]{2}{*}{ Categories } & \multicolumn{4}{|c|}{ Acceptance } & \multicolumn{4}{|c|}{ Humor } & \multicolumn{4}{|c|}{ Religion } \\
\hline & & $\begin{array}{l}\text { Idon't } \\
\text { do this } \\
\text { at all } N\end{array}$ & $\begin{array}{l}\text { I do this a } \\
\text { little bit } \\
N\end{array}$ & $\begin{array}{l}\text { Ido this a } \\
\text { medium } \\
\text { amount } \\
N\end{array}$ & $\begin{array}{c}\text { Ido this a lot } \\
N\end{array}$ & $\begin{array}{l}\text { Idon't } \\
\text { do } \\
\text { this at } \\
\text { all } N\end{array}$ & $\begin{array}{l}\text { Id do this a } \\
\text { little bit } \\
N\end{array}$ & $\begin{array}{c}\text { Ido this a } \\
\text { medium } \\
\text { amount } \\
N\end{array}$ & $\begin{array}{c}\text { Ido this a } \\
\text { lot } \\
N\end{array}$ & $\begin{array}{l}\text { I don't do } \\
\text { this at all } \\
N\end{array}$ & $\begin{array}{l}\text { Id do this a } \\
\text { little bit } \\
N\end{array}$ & $\begin{array}{c}\text { Ido this a } \\
\text { medium } \\
\text { amount } \\
N\end{array}$ & $\begin{array}{c}\text { I do this a } \\
\text { lot } \\
N\end{array}$ \\
\hline \multirow[t]{4}{*}{ Age } & $19-20$ & $2(2)$ & $8(8.33$ & $31(32.29)$ & $15(15.62)$ & $2(2)$ & $22(22.91$ & $26(27.08)$ & $6(6.25)$ & $2(2)$ & $9(9.37)$ & $21(21.87)$ & $24(25)$ \\
\hline & $21-22$ & $0(0)$ & $10(10.41)$ & $17(17.7)$ & $7(7.29)$ & $0(0)$ & $15(15.62)$ & $12(12.5)$ & $7(7.29)$ & $2(2)$ & $8(8.33$ & $11(11.45)$ & $13(13.54)$ \\
\hline & $23 \cdot 24$ & $0(0)$ & $0(0)$ & $2(2)$ & $1(1)$ & $0(0)$ & $2(2)$ & $0(0)$ & $1(1)$ & $0(0)$ & $0(0)$ & $2(2)$ & $1(1)$ \\
\hline & 324 & 1(1) & $1(1)$ & $0(0)$ & $2(2)$ & $0(0)$ & $1(1)$ & $1(1)$ & $2(2)$ & $0(0)$ & $0(0)$ & $2(2)$ & $2(2)$ \\
\hline \multirow[t]{3}{*}{ Family status } & single & $2(2)$ & $16(16.66)$ & $48(50)$ & $22(22.91)$ & $2(2)$ & $35(36.45)$ & $38(39.58)$ & $13(13.54)$ & $3(3)$ & $15(15.62)$ & $32(33.33)$ & $38(39.58)$ \\
\hline & married & 1(1) & $3(3)$ & $1(1)$ & $2(2)$ & $0(0)$ & $4(4.16)$ & $0(0)$ & $3(3)$ & 1(1) & $2(2)$ & $2(2)$ & $2(2)$ \\
\hline & divorce & $0(0)$ & $0(0)$ & $1(1)$ & $0(0)$ & $0(0)$ & $0(0)$ & $1(1)$ & $0(0)$ & $0(0)$ & $0(0)$ & 1(1) & $0(0)$ \\
\hline \multirow[t]{4}{*}{ No. Of children } & Ochild & $2(2)$ & 19(19.79) & $49(51.04)$ & $23(23.95)$ & $2(2)$ & $39(40.62)$ & $39(40.62)$ & $13(13.54)$ & $3(3)$ & $17(17.7)$ & $34(35.41)$ & $39(40.62)$ \\
\hline & Ichild & $1(1)$ & $0(0)$ & $0(0)$ & $1(1)$ & $0(0)$ & $1(1)$ & $0(0)$ & $1(1)$ & $1(1)$ & $0(0)$ & $0(0)$ & $1(1)$ \\
\hline & 2 child & $0(0)$ & $0(0)$ & $0(0)$ & 1(1) & $0(0)$ & $0(0)$ & $0(0)$ & 1(1) & $0(0)$ & $0(0)$ & $1(1)$ & $0(0)$ \\
\hline & 3child & $0(0)$ & $0(0)$ & 1(1) & $0(0)$ & $0(0)$ & $0(0)$ & $0(0)$ & $1(1)$ & $0(0)$ & $0(0)$ & 1(1) & $0(0)$ \\
\hline \multirow[t]{3}{*}{ Year of study } & second year & $3(3)$ & $8(8.33$ & $32(33.33)$ & $16(16.66)$ & $2(2)$ & $22(22.91)$ & $26(27.08)$ & $9(9.37)$ & $2(2)$ & $9(9.37)$ & $24(25)$ & $24(25)$ \\
\hline & third year & $0(0)$ & $7(7.29)$ & $5(5.20)$ & $3(3)$ & $0(0)$ & $8(8.33$ & $66(6.25)$ & $1(1)$ & 1(1) & $4(4.16)$ & $4(4.16)$ & $6(6.25)$ \\
\hline & fourthyear & $0(0)$ & $4(4.16)$ & $13(13.54)$ & $6(6.25)$ & $0(0)$ & $10(10.41)$ & $7(7.29)$ & $66(6.25)$ & $1(1)$ & $4(4.16)$ & $8(8.33)$ & $10(10.41)$ \\
\hline \multirow{2}{*}{$\begin{array}{c}\text { Living with } \\
\text { family }\end{array}$} & yes & $3(3)$ & $17(17.7)$ & $49(51.04$ & $24(25)$ & $2(2)$ & $38(39.58)$ & $39(40.62)$ & $14(14.58)$ & $4(4.16)$ & $16(16.66)$ & $33(34.37)$ & $40(41.66)$ \\
\hline & no & $0(0)$ & $2(2)$ & $1(1)$ & $1(1)$ & $0(0)$ & $2(2)$ & $0(0)$ & $2(2)$ & $0(0)$ & $1(1)$ & $3(3)$ & $0(0)$ \\
\hline \multirow{5}{*}{$\begin{array}{l}\text { Breadwinner } \\
\text { for students }\end{array}$} & father & $1(1)$ & $14(14.5)$ & $42(43.75)$ & $13(13.54)$ & $1(1)$ & $27(28.12)$ & $32(33.33)$ & $10(10.41)$ & $3(3)$ & $12(12.5)$ & $27(28.12)$ & $28(29.16)$ \\
\hline & mother & $0(0)$ & $1(1)$ & $4(4.16)$ & $3(3)$ & $1(1)$ & $3(3)$ & $2(2)$ & $2(2)$ & $0(0)$ & $0(0)$ & $2(2)$ & $66(6.25)$ \\
\hline & brother & $1(1)$ & $0(0)$ & $1(1)$ & $1(1)$ & $0(0)$ & $2(2)$ & $0(0)$ & 1(1) & $0(0)$ & 1(1) & $2(2)$ & $0(0)$ \\
\hline & husband & $1(1)$ & $1(1)$ & 1(1) & $2(2)$ & $0(0)$ & $2(2)$ & $O(0)$ & $3(3)$ & 1(1) & 1(1) & $2(2)$ & $1(1)$ \\
\hline & other & $0(0)$ & $3(3)$ & $2(2)$ & $6(6.25)$ & $0(0)$ & $6(6.25)$ & $5(5.20)$ & $0(0)$ & $0(0)$ & $3(3)$ & $3(3)$ & $5(5.20)$ \\
\hline
\end{tabular}

\begin{tabular}{|c|c|c|c|c|c|c|c|c|c|c|c|c|c|}
\hline \multirow[t]{2}{*}{ Socio - demographic } & \multirow[t]{2}{*}{ Categories } & \multicolumn{4}{|c|}{ Using Emotional Support } & \multicolumn{4}{|c|}{ Self-Distraction } & \multicolumn{4}{|c|}{ Denial } \\
\hline & & $\begin{array}{c}\text { Idont } \\
\text { do } \\
\text { this at } \\
\text { all } N\end{array}$ & $\begin{array}{l}\text { I do this a } \\
\text { little bit } \\
N\end{array}$ & $\begin{array}{c}\text { Ido this a } \\
\text { medium } \\
\text { amount } \\
N\end{array}$ & $\begin{array}{c}\text { I do this a } \\
\text { lot } \\
N\end{array}$ & $\begin{array}{c}\text { Idont do } \\
\text { this at all } \\
N\end{array}$ & $\begin{array}{l}\text { Id do this a } \\
\text { little bit } \\
N\end{array}$ & $\begin{array}{c}\text { Ido this a } \\
\text { medium } \\
\text { amount } \\
N\end{array}$ & $\begin{array}{c}\text { I do this a } \\
\text { lot } \\
N\end{array}$ & $\begin{array}{l}\text { I dont do } \\
\text { this at all } N\end{array}$ & $\begin{array}{l}\text { I do this a } \\
\text { little bit } \\
N\end{array}$ & $\begin{array}{c}\text { Ido this a } \\
\text { medium } \\
\text { amount } \\
N\end{array}$ & $\begin{array}{c}\text { Ido } \\
\text { this a } \\
\text { lot } \\
N\end{array}$ \\
\hline \multirow[t]{4}{*}{ Age } & $19-20$ & $0(0)$ & $22(22.91)$ & $23(23.95)$ & $11(11.45)$ & $5(5.20)$ & $15(15.62)$ & $27(28.12)$ & $9(9.37)$ & $23(23.95)$ & $21(21.87)$ & $10(10.41)$ & $1(1)$ \\
\hline & $21-22$ & $2(2)$ & $11(11.45)$ & $15(15.62)$ & $6(6.25)$ & $1(1)$ & $11(11.45)$ & $16(16.66)$ & $6(6.25)$ & $8(8.33)$ & $18(18.75)$ & $7(7.29)$ & $1(1)$ \\
\hline & 23.24 & $0(0)$ & $1(1)$ & $2(2)$ & $0(0)$ & $0(0)$ & $1(1)$ & $1(1)$ & $1(1)$ & $1(1)$ & $2(2)$ & $0(0)$ & $0(0)$ \\
\hline & 224 & $0(0)$ & $1(1)$ & $1(1)$ & $2(2)$ & $0(0)$ & $0(0)$ & $3(3)$ & $1(1)$ & $0(0)$ & $0(0)$ & $4(4.16)$ & $0(0)$ \\
\hline \multirow[t]{3}{*}{ Family status } & single & $2(2)$ & $31(32.29)$ & $38(39.58)$ & $17(17.7)$ & $5(5.20)$ & $25(26.04)$ & $41(42.7)$ & $17(17.7)$ & $31(32.29)$ & $37(38.54)$ & $18(18.75)$ & $1(1)$ \\
\hline & married & $0(0)$ & $3(3)$ & $2(2)$ & $2(2)$ & $1(1)$ & $1(1)$ & $5(5.20)$ & $0(0)$ & $1(1)$ & $3(3)$ & $2(2)$ & $1(1)$ \\
\hline & divorce & $0(0)$ & $1(1)$ & $0(0)$ & $0(0)$ & $0(0)$ & 1(1) & $0(0)$ & $0(0)$ & $0(0)$ & $1(1)$ & $0(0)$ & $0(0)$ \\
\hline \multirow[t]{4}{*}{ No. Of children } & Ochild & $2(2)$ & $34(35.41)$ & $39(40.62)$ & $18(18.75)$ & $6(6.25)$ & $27(28.12)$ & $43(44.79)$ & $17(17.7)$ & $31(32.29)$ & $41(42.7)$ & $19(19.79)$ & $1(1)$ \\
\hline & Ichild & $0(0)$ & $1(1)$ & $1(1)$ & $0(0)$ & $0(0)$ & $0(0)$ & $2(2)$ & $0(0)$ & $1(1)$ & $0(0)$ & $1(1)$ & $0(0)$ \\
\hline & 2 child & $0(0)$ & $0(0)$ & $0(0)$ & $1(1)$ & $0(0)$ & $0(0)$ & $1(1)$ & $0(0)$ & $0(0)$ & $0(0)$ & $1(1)$ & $0(0)$ \\
\hline & 3child & $0(0)$ & $0(0)$ & $1(1)$ & $0(0)$ & $0(0)$ & $0(0)$ & $1(1)$ & $0(0)$ & $0(0)$ & $0(0)$ & $0(0)$ & $1(1)$ \\
\hline \multirow[t]{3}{*}{ Year of study } & second year & $0(0)$ & $22(22.91)$ & $27(28.12)$ & $10(10.41)$ & $5(5.20)$ & $15(15.62)$ & $28(29.16)$ & $11(11.45)$ & $22(22.91)$ & $21(21.87)$ & $13(13.54)$ & $2(2)$ \\
\hline & third year & $1(1)$ & $7(7.29)$ & $5(5.20)$ & $2(2)$ & $0(0)$ & $5(5.20)$ & $8(8.33)$ & $2(2)$ & $3(3)$ & $8(8.33)$ & $4(4.16)$ & $0(0)$ \\
\hline & fourth year & 1(1) & $6(6.25)$ & $9(9.37)$ & $7(7.29)$ & $1(1)$ & $7(7.29)$ & $11(11.45)$ & $4(4.16)$ & $7(7.29)$ & $12(12.5)$ & $4(4.16)$ & $0(0)$ \\
\hline \multirow[t]{2}{*}{ Living with family } & yes & $2(2)$ & $34(35.41)$ & $38(39.58)$ & 19(19.79) & $4(4.16)$ & $26(27.08)$ & $46(47.91)$ & $17(17.7)$ & $30(31.25)$ & $40(41.60)$ & $21(21.87)$ & $1(1)$ \\
\hline & no & $0(0)$ & $1(1)$ & $3(3)$ & $0(0)$ & $2(2)$ & $1(1)$ & $1(1)$ & $0(0)$ & $2(2)$ & $1(1)$ & $0(0)$ & $1(1)$ \\
\hline \multirow{5}{*}{$\begin{array}{l}\text { Breadruinner for } \\
\text { students }\end{array}$} & father & $2(2)$ & $25(26.04)$ & $29(30.20)$ & $14(14.58)$ & $3(3)$ & $23(23.95)$ & $30(31.25)$ & $14(14.58)$ & $23(23.95)$ & $32(33.33)$ & $14(14.58)$ & $1(1)$ \\
\hline & mother & $0(0)$ & $1(1)$ & $66(6.25)$ & $1(1)$ & $0(0)$ & $2(2)$ & $3(3)$ & $3(3)$ & $3(3)$ & $3(3)$ & $1(1)$ & $0(0)$ \\
\hline & brother & $0(0)$ & $2(2)$ & $1(1)$ & $0(0)$ & $0(0)$ & $0(0)$ & $3(3)$ & $0(0)$ & $0(0)$ & $2(2)$ & 1(1) & $0(0)$ \\
\hline & husband & $0(0)$ & $2(2)$ & $2(2)$ & 1(1) & $0(0)$ & $1(1)$ & $4(4.16)$ & $0(0)$ & $1(1)$ & $1(1)$ & $2(2)$ & $1(1)$ \\
\hline & other & $0(0)$ & $5(5.20)$ & $3(3)$ & $3(3)$ & $3(3)$ & $1(1)$ & $7(7.29)$ & $0(0)$ & $5(5.20)$ & $3(3)$ & $3(3)$ & $0(0)$ \\
\hline \multirow[t]{3}{*}{ Socio - demographic } & Categories & \multirow{2}{*}{\multicolumn{4}{|c|}{ Venting }} & \multicolumn{4}{|c|}{ Substance Use } & \multirow{2}{*}{\multicolumn{4}{|c|}{ Behavioral Disengagement }} \\
\hline & & & & & & I don't do & I do this & Ido this a & Id do this a & Idon't do & I do this a & Ido this a & \\
\hline & & $\begin{array}{c}\text { this at all } \\
N\end{array}$ & $\begin{array}{l}\text { little bit } \\
N\end{array}$ & $\begin{array}{l}\text { medium } \\
\text { amount } \\
N\end{array}$ & ${ }_{N}^{\text {lot }}$ & this at all $N$ & $\begin{array}{l}\text { a little } \\
\text { bit } \\
N\end{array}$ & $\begin{array}{l}\text { medium } \\
\text { amount }\end{array}$ & $\begin{array}{l}\text { lot } \\
N\end{array}$ & this at all $N$ & $\begin{array}{l}\text { little bit } \\
N\end{array}$ & $\begin{array}{l}\text { medium } \\
\text { amount }\end{array}$ & a lot \\
\hline \multirow[t]{4}{*}{ Age } & $19-20$ & $3(3)$ & $21(21.6)$ & $23(23.95)$ & $9(9.37)$ & $51(53.12)$ & $3(3)$ & $1(1)$ & $51(53.12$ & $12(12.5)$ & $33(34.37$ & $11(11.45)$ & $0(0)$ \\
\hline & $21-22$ & $2(2)$ & $14(14.4)$ & $17(17.7)$ & $1(1)$ & $25(26.04)$ & $5(5.20)$ & $4(4.16)$ & $25(26.04)$ & $8(8.33)$ & $14(14.4)$ & $8(8.33)$ & $4(4.16)$ \\
\hline & $23-24$ & $0(0)$ & $2(2)$ & $0(0)$ & $1(1)$ & $3(3)$ & $0(0)$ & $0(0)$ & $3(3)$ & $0(0)$ & $3(3)$ & $0(0)$ & $0(0)$ \\
\hline & $>24$ & $0(0)$ & $1(1)$ & $3(3)$ & $0(0)$ & $\frac{4(4.16)}{4(6)}$ & $0(0)$ & & $\frac{4(4.16)}{4}$ & $0(0)$ & $3(3)$ & $1(1)$ & $0(0)$ \\
\hline \multirow[t]{3}{*}{$\begin{array}{l}\text { Family status } \\
\end{array}$} & single & $5(5.20)$ & $34(35.41)$ & $39(40.62)$ & $10(10.41)$ & $77(80.2)$ & $6(6.25)$ & $4(4.16)$ & $77(80.2)$ & 19(19.79) & $51(53.12)$ & $16(16.66)$ & $2(2)$ \\
\hline & & $0(0)$ & $4(4.16)$ & $3(3)$ & $0(0)$ & $4(4.16)$ & $2(2)$ & $1(1)$ & $4(4.16)$ & $0(0)$ & $2(2)$ & $3(3)$ & $2(2)$ \\
\hline & & $0(0)$ & & $1(1)$ & $0(0)$ & $1(1)$ & $0(0)$ & $0(0)$ & $1(1)$ & $1(1)$ & $0(0)$ & $0(0)$ & $0(0)$ \\
\hline \multirow[t]{4}{*}{ No. Of children } & & $5(5.20)$ & $35(36.45)$ & $42(43.75)$ & $11(11.45)$ & $80(83.33)$ & $7(7.29)$ & $5(5.20)$ & $80(83.33)$ & $20(20.83)$ & $52(54.16)$ & $18(18.75)$ & $3(3)$ \\
\hline & & $0(0)$ & $1(1)$ & $1(1) 1$ & $0(0)$ & $2(2)$ & $0(0)$ & $0(0)$ & $2(2)$ & $0(0)$ & $0(0)$ & $2(2)$ & $0(0)$ \\
\hline & 2 child & $0(0)$ & $1(1)$ & $0(0)$ & $0(0)$ & $1(1)$ & $0(0)$ & $0(0)$ & $1(1)$ & $0(0)$ & 1(1) & $0(0)$ & $0(0)$ \\
\hline & 3child & $0(0)$ & & $0(0)$ & $0(0)$ & & $1(1)$ & $0(0)$ & $0(0)$ & $0(0)$ & $0(0)$ & $0(0)$ & $1(1)$ \\
\hline \multirow[t]{3}{*}{$\begin{array}{l}\text { Year of study } \\
\end{array}$} & second year & $3(3)$ & $22(22.91)$ & $26(27.08)$ & $8(8.33)$ & $53(55.2)$ & $3(3)$ & $2(2)$ & $53(55.2)$ & $12(12.5)^{2}$ & $34(35.41)$ & $12(12.5)$ & $1(1)$ \\
\hline & third year & $1(1)$ & $99(9.37)$ & $4(4.16)$ & $1(1)$ & $11(11.45)$ & $3(3)$ & 1(1) & $11(11.45)$ & $6(6.25)$ & $5(5.20)$ & $4(4.16)$ & $0(0)$ \\
\hline & fourth year & $1(1)$ & $7(7.29)$ & $13(13.54)$ & & 19(19.79) & $2(2)$ & $2(2)$ & 19(19.79) & $2(2)$ & $14(14.58)$ & $4(4.16)$ & $3(3)$ \\
\hline Living with family & & $5(5.20)$ & $37(38.54)$ & & $11(11.45)$ & $81(84.37)$ & $7(7.29)$ & $4(4.16)$ & $0(0)$ & $20(20.83)$ & $51(53.12)$ & $20(20.83)$ & $2(2)$ \\
\hline & & & & & of & & $1(1)$ & $1(1)$ & $0(0)$ & $0(0)$ & $2(2)$ & $0(0)$ & $2(2)$ \\
\hline Breadwinner for & father & $4(4.16)$ & $29(30.2)$ & $31(32.29)$ & $6(6.25)$ & $59(61.45)$ & $6(6.25)$ & $4(4.16)$ & $0(0)$ & $15(15.62)$ & $39(40.62)$ & $15(15.62)$ & $1(1)$ \\
\hline students & mother & $0(0)$ & $2(2)$ & $4(4.16)$ & $2(2)$ & $8(8.33)$ & $0(0)$ & $0(0)$ & $0(0)$ & $2(2)$ & $5(5.20)$ & $0(0)$ & $1(1)$ \\
\hline & brother & 1(1) & $0(0)$ & $2(2)$ & $0(0)$ & $3(3)$ & $0(0)$ & $0(0)$ & $0(0)$ & $0(0)$ & $2(2)$ & $1(1)$ & $0(0)$ \\
\hline & husband & $0(0)$ & $4(4.16)$ & $1(1)$ & $0(0)$ & $3(3)$ & $2(2)$ & $0(0)$ & $0(0)$ & $0(0)$ & $2(2)$ & $2(2)$ & $1(1)$ \\
\hline & other & $0(0)$ & $3(3)$ & $5(5.20)$ & $3(3)$ & $10(10.41)$ & $0(0)$ & $1(1)$ & $0(0)$ & $3(3)$ & $5(5.20)$ & $2(2)$ & 1(1) \\
\hline
\end{tabular}


Perceived Level Of Stress, \& Coping Strategies Among Saudi Nursing Student

\begin{tabular}{|c|c|c|c|c|c|c|c|c|c|}
\hline \multirow[t]{2}{*}{ Socio - demographic } & \multirow[t]{2}{*}{ Categories } & \multicolumn{4}{|c|}{ Self-Blame } & \multicolumn{4}{|c|}{ Using Instrumental Support } \\
\hline & & $\begin{array}{l}\text { I don't do } \\
\text { this at all } N\end{array}$ & $\begin{array}{c}\text { I do this a } \\
\text { little bit } \\
N\end{array}$ & $\begin{array}{l}\text { Ido this a } \\
\text { medium } \\
\text { amount } \\
N\end{array}$ & $\begin{array}{c}\text { I do this a lot } \\
\qquad N\end{array}$ & $\begin{array}{l}\text { I don't do } \\
\text { this at all } N\end{array}$ & $\begin{array}{c}\text { I do this a } \\
\text { little bit } \\
N\end{array}$ & $\begin{array}{c}\text { Ido this a } \\
\text { medium } \\
\text { amount } \\
N\end{array}$ & $\begin{array}{c}\text { I do this a lot } \\
\qquad N\end{array}$ \\
\hline \multirow[t]{4}{*}{ Age } & $19-20$ & $5(5.20)$ & $17(17.7)$ & $24(25)$ & $10(10.41)$ & $5(5.20)$ & $15(15.62)$ & 19(19.79) & $17(17.7)$ \\
\hline & $21-22$ & $3(3)$ & $12(12.5)$ & $15(15.62)$ & $4(4.16)$ & $2(2)$ & $11(11.45)$ & $14(14.58)$ & $7(7.29)$ \\
\hline & $23-24$ & $0(0)$ & $0(0)$ & $1(1)$ & $2(2)$ & $0(0)$ & $0(0)$ & $1(1)$ & $2(2)$ \\
\hline & $>24$ & $0(0)$ & $0(0)$ & $3(3)$ & $1(1)$ & $0(0)$ & $0(0)$ & $3(3)$ & $1(1)$ \\
\hline \multirow[t]{3}{*}{ Family status } & single & $8(8.33)$ & $27(28.12)$ & $37(38.54)$ & $16(16.66)$ & $7(7.29)$ & $24(25)$ & $33(34.37)$ & $24(25)$ \\
\hline & married & $0(0)$ & $2(2)$ & $5(5.20)$ & $0(0)$ & $0(0)$ & $2(2)$ & $3(3)$ & $2(2)$ \\
\hline & divorce & $0(0)$ & $0(0)$ & $0(0)$ & 1(1) & $0(0)$ & $0(0)$ & $0(0)$ & $1(1)$ \\
\hline \multirow[t]{4}{*}{ No. Of children } & Ochild & $8(8.33)$ & $29(30.2)$ & $39(40.62)$ & $17(17.7)$ & $7(7.29)$ & $26(27.08)$ & $34(35.41)$ & $26(27.08)$ \\
\hline & Ichild & $0(0)$ & $0(0)$ & $2(2)$ & $0(0)$ & $0(0)$ & $0(0)$ & $2(2)$ & $0(0)$ \\
\hline & 2 child & $0(0)$ & $0(0)$ & 1(1) & $0(0)$ & $0(0)$ & $0(0)$ & $1(1)$ & $0(0)$ \\
\hline & 3child & $0(0)$ & $0(0)$ & $1(1)$ & $0(0)$ & $0(0)$ & $0(0)$ & $0(0)$ & $1(1)$ \\
\hline \multirow[t]{3}{*}{ Year of study } & second year & $5(5.20)$ & $17(17.7)$ & $28(29.16)$ & $9(9.37)$ & $5(5.20)$ & $16(16.66)$ & $21(21.87)$ & $17(17.7)$ \\
\hline & third year & $2(2)$ & $5(5.20)$ & $6(6.25)$ & $2(2)$ & $1(1)$ & 4(4.16) & $7(7.29)$ & $3(3)$ \\
\hline & fourth year & $1(1)$ & $7(7.29)$ & $9(9.37)$ & $6(6.25)$ & $1(1)$ & $6(6.25)$ & $9(9.37)$ & $7(7.29)$ \\
\hline \multirow[t]{2}{*}{ Living with family } & yes & $7(7.29)$ & $28(29.16)$ & $41(42.7)$ & $17(17.7)$ & $7(7.29)$ & $25(26.04)$ & $35(36.45)$ & $26(27.08)$ \\
\hline & no & $1(1)$ & $1(1)$ & $2(2)$ & $0(0)$ & $0(0)$ & $1(1)$ & $2(2)$ & $1(1)$ \\
\hline \multirow{5}{*}{$\begin{array}{l}\text { Breadwinner for } \\
\text { students }\end{array}$} & father & $6(6.25)$ & $24(25)$ & $26(27.08)$ & $14(14.58)$ & $5(5.20)$ & $20(20.83)$ & $26(27.08)$ & 19(19.79) \\
\hline & mother & $1(1)$ & $2(2)$ & 4(4.16) & 1(1) & $1(1)$ & $2(2)$ & $3(3)$ & $2(2)$ \\
\hline & brother & $0(0)$ & $0(0)$ & $2(2)$ & 1(1) & $0(0)$ & $0(0)$ & $2(2)$ & 1(1) \\
\hline & husband & $0(0)$ & $1(1)$ & $4(4.16)$ & $0(0)$ & $0(0)$ & 1(1) & $3(3)$ & 1(1) \\
\hline & other & $1(1)$ & $2(2)$ & $7(7.29)$ & $1(1)$ & $1(1)$ & $3(3)$ & $3(3)$ & $4(4.16)$ \\
\hline
\end{tabular}

Table (4) The relation between the coping strategies \& stressors.

\begin{tabular}{|c|c|c|c|c|c|c|c|c|c|}
\hline \multirow{3}{*}{$\begin{array}{c}\text { Socio- } \\
\text { demographic }\end{array}$} & \multirow{3}{*}{ Categories } & \multicolumn{8}{|c|}{ Copies strategies used by nursing student (Adaptive Coping) } \\
\hline & & active coping & planning & positive reframing & acceptance & humor & religion & $\begin{array}{l}\text { using emotional } \\
\text { support }\end{array}$ & $\begin{array}{l}\text { using instrumental } \\
\text { support }\end{array}$ \\
\hline & & \multicolumn{8}{|c|}{ Chi square $P$-value } \\
\hline \multirow[t]{4}{*}{ Age } & $19-20$ & \multirow[t]{4}{*}{.237} & \multirow[t]{4}{*}{.141} & \multirow[t]{4}{*}{.453} & \multirow[t]{4}{*}{.114} & \multirow[t]{4}{*}{.418} & \multirow[t]{4}{*}{918} & \multirow[t]{4}{*}{.592} & \multirow[t]{4}{*}{.609} \\
\hline & $21-22$ & & & & & & & & \\
\hline & $23-24$ & & & & & & & & \\
\hline & $>24$ & & & & & & & & \\
\hline \multirow{3}{*}{ Family status } & single & \multirow[t]{3}{*}{962} & \multirow[t]{3}{*}{.196} & \multirow[t]{3}{*}{.224} & \multirow[t]{3}{*}{240} & \multirow[t]{3}{*}{226} & \multirow[t]{3}{*}{.605} & \multirow[t]{3}{*}{.853} & \multirow[t]{3}{*}{.783} \\
\hline & married & & & & & & & & \\
\hline & divorce & & & & & & & & \\
\hline \multirow{4}{*}{ No. Of children } & Ochild & \multirow{4}{*}{.812} & \multirow{4}{*}{.258} & \multirow{4}{*}{.726} & \multirow{4}{*}{$.016^{*}$} & \multirow{4}{*}{.171} & \multirow{4}{*}{.092} & \multirow{4}{*}{.734} & \multirow{4}{*}{.577} \\
\hline & Ichild & & & & & & & & \\
\hline & 2 child & & & & & & & & \\
\hline & 3 child & & & & & & & & \\
\hline \multirow[t]{3}{*}{ Year of study } & secondyear & .869 & .405 & .772 & .121 & .537 & 927 & .333 & .977 \\
\hline & third year & & & & & & & & \\
\hline & fourth year & & & & & & & & \\
\hline Living with & yes & .621 & .526 & .922 & .445 & .197 & .303 & .551 & .925 \\
\hline family & no & & & & & & & & \\
\hline Breadwinner for & father & .903 & .101 & .125 & $.010^{*}$ & .095 & .458 & .865 & .983 \\
\hline students & mother & & & & & & & & \\
\hline & brother & & & & & & & & \\
\hline & husband & & & & & & & & \\
\hline & other & & & & & & & & \\
\hline
\end{tabular}

$\mathrm{p}^{*}<0.05$, significant

\begin{tabular}{|c|c|c|c|c|c|c|c|}
\hline \multirow[t]{3}{*}{ Socio - demographic } & \multirow[t]{3}{*}{ Categories } & \multicolumn{6}{|c|}{ Copies strategies used by nursing student ( Maladaptive Coping ) } \\
\hline & & self-distraction & denial & venting & substance use & behavioral disengagement & self-blame \\
\hline & & \multicolumn{6}{|c|}{ Chi square $P$-value } \\
\hline \multirow[t]{4}{*}{ Age } & $19-20$ & \multirow[t]{4}{*}{.878} & \multirow{4}{*}{$.024^{*}$} & \multirow{4}{*}{.459} & \multirow{4}{*}{.222} & \multirow[t]{4}{*}{.182} & \multirow[t]{4}{*}{.445} \\
\hline & $21-22$ & & & & & & \\
\hline & $23-24$ & & & & & & \\
\hline & $>24$ & & & & & & \\
\hline \multirow[t]{3}{*}{ Family status } & single & \multirow[t]{3}{*}{.422} & \multirow[t]{3}{*}{.260} & \multirow[t]{3}{*}{.807} & \multirow[t]{3}{*}{.225} & \multirow[t]{3}{*}{$.004 *$} & \multirow[t]{3}{*}{.250} \\
\hline & married & & & & & & \\
\hline & divorce & & & & & & \\
\hline \multirow[t]{4}{*}{ No. Of children } & Ochild & \multirow[t]{4}{*}{.880} & \multirow[t]{4}{*}{$.000^{*}$} & \multirow[t]{4}{*}{.935} & \multirow[t]{4}{*}{.073} & \multirow[t]{4}{*}{$.000^{*}$} & \multirow[t]{4}{*}{.813} \\
\hline & Ichild & & & & & & \\
\hline & 2 child & & & & & & \\
\hline & 3child & & & & & & \\
\hline \multirow{3}{*}{ Year of study } & second year & \multirow[t]{3}{*}{.903} & \multirow[t]{3}{*}{.627} & \multirow[t]{3}{*}{.573} & \multirow[t]{3}{*}{.332} & \multirow[t]{3}{*}{.060} & \multirow[t]{3}{*}{.857} \\
\hline & third year & & & & & & \\
\hline & fourth year & & & & & & \\
\hline \multirow[t]{2}{*}{ Living with family } & yes & $.003^{*}$ & $.007^{*}$ & .618 & .075 & $.000^{*}$ & .529 \\
\hline & no & & & & & & \\
\hline Breadwinner for & father & $.043^{*}$ & .342 & .177 & .285 & .461 & .735 \\
\hline students & mother & & & & & & \\
\hline & brother & & & & & & \\
\hline & husband & & & & & & \\
\hline & other & & & & & & \\
\hline
\end{tabular}

$\mathrm{p}^{*}<0.05$, significant 


\subsection{The relation between the coping strategies \& stressors: Through reviewing the results illustrated in the table 4 ,it shows :}

That there is a significant correlation between the using of acceptance as copy strategy and Breadwinner \& no.Of children for students $(\mathrm{p}=0.010) \&(\mathrm{p}=0.016)$ respectively . Also ,there is a significant correlation between the using of denial and age $(p=0.024)$, no. Of children for student $(p=0.000) \&$ living with family ( $p=0.007$ ). In addition, there is a significant correlation between the using of behavior disengagement \& family status $(\mathrm{p}=0.004)$, no. Of children for student $(\mathrm{p}=0.000) \&$ living with family $(\mathrm{p}=0.000)$.the result also revealed that there is a significant correlation between the using of self distraction and living with family \&Breadwinner $(p=0.003),(p=0.043)$ respectively. While the results indicates that there is no correlation between the copies strategies using from nursing students ( active coping ,planning, positive reframing, humor, religion, using emotional support, venting ,substances use, self blame, using instrument support ) and stressors (Socio Demographic Changes).

\section{Discussion}

Through a review of previous results showing that Stress in nursing students is an area of growing concern and it may result in psychological distress, physical complaints, behavior problem and poor academic performance. The present study was conducted in one of the governmental nursing college situated in Makkah Al Mukaramh city and affiliated to Umm Al Qura University. Table (1),(2)depicts the relationship of stress level of nursing students to the demographic characteristics. It shows that stress level of nursing students is found $87.0 \%$ of fourth year nursing student has moderate stress. This is due to, students were surveyed near the end of the academic year, just prior to final exams, and this timing may have influenced their responses. These findings confirm previous research, which found that academic related stressors, such as taking tests, writing papers, grades, too little time, and the constant pressure of studying, were the most irritating to nursing and other college students in the United States and other countries. ${ }^{[26,27,28,29, \& 30]}$ Oermann also found that stress experienced by nursing students in clinical practice increased as they progressed through the program. ${ }^{[31]}$ Also, These findingsare in keeping with the results obtained by previous authors. ${ }^{[32,33834]}$ And also, keeping the result with Filipino study which was reported that Filipino students nurses have moderate stress. ${ }^{[35]}$ Moreover, there is a significant correlation between the nursing students' stress level and Breadwinner for students ( $p=0.032$ ).No studies have explored student breadwinner. Finding revealed in figure (2) that nursing students used most Adaptive coping style include religion, acceptance, active coping positive reframing\& humor. Whereas nursing students used maladaptive coping in moderate doing of self distraction \& a little bit of denial. Also the result show that $85.6 \%$ of nursing student don't do the substance use as copy strategy, that means the least of using maladaptive coping style among nursing student .This is similar to Saudi study among residents student it is show that adaptive stress-coping strategies were more frequently used among a sample of residents in Saudi Arabia than maladaptive stress-coping strategies. Religion, planning, acceptance, and active-coping were common adaptive stress-coping strategies, while self-blame, self-distraction, and venting were common maladaptive stress-coping strategies. ${ }^{[36]}$ That mean nursing student satisfy for studying nursing program .This result is conducted with Brasília study that show students satisfied with the course used positive coping strategies targeting the problem, whereas dissatisfied students used negative strategies focusing on the emotion. ${ }^{[37]}$ Table (4) illustrate that The majority of maladaptive stress-coping strategies in the current study were associated demographic data (stressors) including, the using of denial and age $(p=0.024)$,no. Of children for student $(p=0.000) \&$ living with family ( $p=0.007)$. In addition ,the using of behavior disengagement \& family status $(p=0.004)$, no. Of children for student $(p=0.000) \&$ living with family ( $\mathrm{p}=0.000$ ) . The result also revealed that there is a significant correlation between the using of self distraction and living with family \&Breadwinner $(\mathrm{p}=0.003),(\mathrm{p}=0.043)$ respectively .Also there is a significant correlation between adaptive coping style \& stressors such as, the using of acceptance as copy strategy and Breadwinner \& no. Of children for students $(\mathrm{p}=0.010) \&(\mathrm{p}=0.016)$ respectively . whereas, religion was the most frequently used adaptive stress-coping strategy in the current study. This may reflect the critical role of religion in all aspects of behavior in a conservative country such as Saudi Arabia.

\section{Conclusion}

Moderate stress level \& adaptive stress-coping strategies were more frequently used among a sample of nursing student at umm al Qura university in Saudi Arabia .

\section{Recommendation}

It is important for nurse educator to find out the sources of stress and coping strategies used by the students so that they can be helped to cope well with upcoming problems and situations.

\section{Acknowledgements}

The author would like to thank all students who participated in the study.Also, the author acknowledges the Deanship of Scientific Research \& Graduate Student at Umm Al Qura University for supporting this study. 


\section{References}

[1]. J.M. Kohler, D.C. Munz, M.J. Grawitch . Test of a dynamic stress model for organizational change: do males and females require different models? ApplPsycholInt Rev, 55 (2) (2006), pp. 168-191

[2]. M. Pulido-Martos, J.M. Augusto-Landa, E. Lopez-Zafra. Sources of stress in nursing students: a systematic review of quantitative studies. IntNurs Rev, 59 (1) (2011), pp. 15-25

[3]. S. Pryjmachuk, D.A. Richards. Mental health nursing students differ from other nursing students: some observations from a study on stress and coping. Int J Ment Health Nurs, 16 (6) (2007), pp. 390-402.

[4]. F. Timmins, M. Kaliszer. Aspects of nurse education programmes that frequently cause stress to nursing students - fact finding sample survey. $\square$ Nurse Educ Today, 22 (2002), pp. 203-211

[5]. I. Deary, R. Watson, R. Hogston. A longitudinal cohort study of burnout and attrition in nursing students. J AdvNurs, 43 (1) (2003), pp. 71-81.

[6]. H. Brown, R. Edelmann, Project 2000: a study of expected and experienced stressors and support reported by students and qualified nurses. J AdvNurs, 31 (4) (2000), pp. 857-864.

[7]. Güler, Ö. veÇınar, S. (2010). HemşireliköğrencilerininalgıladıklarıstresörlervekullandıklarıbaşetmeyöntemlerininBelirlenmesi. MaltepeÜniversitesiHemşirelikBilimveSanatıDergisi [SempozyumÖzelSayısı], 3, 253-260.

[8]. Martos, M. P., Landa, A., \&Zafra, E. L. (2011). Sources of stress in nursing students: A systematic review of quantitative studies. International Nursing Review, 59, 15-25.

[9]. Özkan, S. ve Yılmaz, E. (2010). Üniversiteöğrencilerininüniversiteortamınauyumdurumları (BandırmaÖrneği). FiratSağlıkHizmetleriDergisi, 5 (13), 153-170.

[10]. Öztürk Can, H., Öner, Ö. İ. veÇelebi, E. (2009). Üniversiteöğrencilerindeeğitiminsorunçözmebecerisineetkisininincelenmesi. FuratSağlıkHizmetleriDergisi, 4 (10), 35-57.

[11]. Seyedfatemi, N., Tafreshi, M., \&Hagani, H. (2007). Experienced stressors and coping strategies among Iranian nursing students. Biomedical Nursing, 6 (11), 1-10.

[12]. Sheu, S., Lin, H. S., \& Hwang, S. (2002). Perceived stres and physio-psycho-social status of nursing students during their initial period of clinical practice: The effect of coping behaviors. International Journal of Nursing Studies, 39, 165-175.

[13]. Warbah, L., Sathiyaseelan, M., Vijayakumar, C., Vasantharaj, B., Russell, S., Jacob, K. S. (2007). Psychological distress, personality, and adjustment among nursing students. Nurse Education Today, 27, 597-601.

[14]. Edwards, D., Burnard, P., Bennett, K., \&Hebden, U. (2010). A longitudinal study of stress and self-esteem in student nurses. Nurse Education Today, 30, 78-84.

[15]. Temel, E., Bahar, A. veÇuhadar, D. (2007). Öğrencihemşirelerinstreslebaşetmetarzlarıvedepresyondüzeylerininbelirlenmesi. FıratSağlıkHizmetleriDergisi, 2 (5), 117-118.

[16]. Ya -T.T.Yang. Stress, Coping, and Psychological Well-Being: Comparison among American and Asian International Graduate Students from Taiwan, China, and South Korea . Date approved: July 16, 2010.

[17]. Pashtoon,M.K.,Haider A.N. ,Abaseen K.A.,Talhak.,FarooqH.K.,Umber Z.K.,Urooj B.K.,Jawadk.,\&Hadi M.K. Coping Styles In Patients With Anxiety And Depression .ISRN Psychiatry ,2012 vol.2012, ID128672 ,7 pages. http://dx.doi.org/10.5402/2012/128672

[18]. H. Nawel, S. Elisabeth Adaptation and validation of the Tunisian version of the Brief COPE Scale. EHP vol.17 supp.2015.

[19]. N Yusoff; WY Low; CH Yip .Reliability And Validity Of The Malay Version Of Brief Cope Scale: A Study On Malaysian Women Treated With Adjuvant Chemotherapy For Breast Cancer . MJP Online Early 01-04-09.

[20]. N Yusoff W Y Low, C H Yip. Reliability and Validity of the Brief COPE Scale (English Version) Among Women with Breast Cancer Undergoing Treatment of Adjuvant Chemotherapy: A Malaysian Study.Med J Malaysia Vol. 65 No. 1 March 2010.

[21]. The PSS Scale is reprinted with permission of the American Sociological Association, from Cohen, S., Kamarck, T., and Mermelstein, R..Aglobal measure of perceived stress. Journal of Health and Social Behavior, 1983,24, 386-396.

[22]. Cohen, S. and Williamson, G. Perceived Stress in a Probability Sample of the United States. Spacapan, S. and Oskamp, S. (Eds.) The SocialPsychology of Health. Newbury Park, CA: Sage, 1988.

[23]. Eun-Hyun Lee .Review Of Psychometric Evidence Of The Percived Stress Scale .Asian Nursing Research .2012;Vol.6(4) : 121-127 .http://www.friendsnrc.org/. National Resource Center .February 2006.

[24]. Almadi T, Cathers I, Hamdan Mansour AM, Chow CM.An Arabic version of the perceived stress scale: translation and validation study. Int J Nurs Stud. 2012 Jan;49(1):84-9. doi: 10.1016/j.ijnurstu.2011.07.012. Epub 2011 Aug 17.

[25]. Beck, D. L., Hackett, M. B., Srivastava, R., McKim, E., \& Rockwell, B. (1997). Perceived level and sources of stress in university professional schools. Journal of Nursing Education, 36(4), 180-186.

[26]. Beck, D. L., \&Srivastava, R. (1991). Perceived level and sources of stress in baccalaureate nursing students. Journal of Nursing Education, 30(3), 127-133.

[27]. Gadzella, B. M., Masten, W., \& Stacks, J. (1998). Students' stress and their learning strategies, test anxiety, and attributions. College Student Journal, 32(3), 416-422.

[28]. Schafer, W. (1996). Passing the test of college stress. In W. Schafer (Ed.), Stress management for wellness (pp. 543-563). Orlando: Harcourt Brace.

[29]. Robotham, D., \& Julian, C. (2006). Stress and the higher education student: A critical review of the literature. Journal of Further and Higher Education, 30(2), 107-117.

[30]. Oermann M.H. Differences in clinical experiences of ADN and BSN students. Journal of Nursing Education. 1998; 37(5):197-201. PMid:9605192.

[31]. Chan KL, So KW, Fong YT. Hong Kong baccalaureate nursing students' stress and their coping strategies in clinical practice. Journal of Professional Nursing 2009;25(5), 307-313.http://dx.doi.org/10.1016/j.profnurs.2009.01.018 PMid:19751936

[32]. Pagana KD. Stress and threats reported by baccalaureate students in relation to an initial clinical experience. Journal of Nursing Education 1988; 27(9), 418-424.PMid:2852715.

[33]. Sharma N, Kaur A. Factors associated with stress among nursing students. Nursing and Midwifery Research Journal 2011; 7(1), $12-21$.

[34]. Leodoro J. L. .Stress, Stressors, and Stress Responses of Student Nurses in a Government Nursing School .Health science journal. Volume 7 (2013),Issue 4

[35]. Fahad D. Alosaimi, ${ }^{1}$ Auroabah Almufleh, ${ }^{2}$ Sana Kazim, ${ }^{3}$ and Bandar Aladwani. Stress-coping strategies among medical residents in Saudi Arabia: A cross-sectional national study.Pak J Med Sci. 2015 May-Jun; 31(3): 504-509. doi: 10.12669/pjms.313.7490

[36]. Carolina D .H. ,EdisonL.B., LedaK.A.,JamilaG.B,AlineB.F., ValériaL.L. Coping strategies of nursing students for dealing with university stress. Enferm. vol.68 no.5 Rev. Bras. Brasília Sept./Oct. 2015 . http://dx.doi.org/10.1590/0034-7167.2015680503i. 